\title{
Questions on accreting mass and minimum magnetic field of millisecond pulsars
}

\author{
C. M. Zhang \\ National Astronomical Observatories, Chinese Academy of Sciences, Beijing 100012, China \\ email: zhangcm@bao.ac.cn
}

\begin{abstract}
About 0.2 solar mass is absorbed by the millisecond pulsar (MSP) at the binary accretion phase, while the polar magnetic field of MSP is diluted to a magnitude of order $10^{8.5}$ Gauss, which is proportionally related to the mass accretion rate. It is found that the minimum magnetic field of MSP can be as low as $10^{7}$ Gauss if the accretion rate of the binary system reaches its the minimum value of $10^{15} \mathrm{~g} / \mathrm{s}$. This bottom field has nothing to do with the MSP initial field. Some questions on MPSs are proposed and answered.
\end{abstract}

Keywords. Pulsar, neutron star, mass, magnetic field

\section{General picture of MSP evolution}

The millisecond pulsar (MSP) is formed in the binary accretion phase, while the neutron star (NS) accretes matter from its companion. The magnetic field and spin period are both decreased to the values of $10^{8.5}$ Gauss and several milliseconds (Wijnands \& van der Klis 1998), if the system absorbed the mass of about 0.2 solar mass (Bhattacharya \& van den Heuvel 1991; Wang et al. 2011; Zhang et al. 2011; Alpar et al. 1982; Tauris 2012). Then the conventional NS is formed from the supernova explosion, while a high magnetic field of about $10^{11-13} \mathrm{G}$ and spin period of about 20-30 milliseconds, which can be seen from the observed pulsar data of ATNF (Manchester et al. 2005). In a NS binary system, if the accretion mass of $\sim 0.01 M_{\odot}$ is accreted from the companion, a NS can be spun up to several tens of milliseconds, while its magnetic field will be decayed to $\sim 10^{9-10} \mathrm{G}$, e.g. the double pulsar system (Lyne et al. 2004; van den Heuvel 2004). Therefore, more mass accreted and less magnetic field of NS achieved (Zhang \& Kojima 2006). In this short paper we present some enquiries and basic conclusions on MSP evolution.

\section{The bottom field and minimum field of MSP}

During the accretion phase, if the NS field is high, then the accreting MHD materials flow along the polar field lines to fall on the NS surface, while the MHD matter is piled-up at the magnetic polar cap and it flows from the polar cap to the equator, while the MHD flow drags the field lines asides to dilute the polar field strength (Zhang \& Kojima 2006). The accretion flow continues until the field lines are all trapped into the star, at where the magnetosphere of NS equals to the NS radius, which can give a bottom field $\left(B_{f}\right)$ of NS of about $\sim 10^{8.5} \mathrm{G}$ after the system accretes about 0.2 solar mass,

$$
B_{f} \simeq 10^{8.5}(G)\left(\dot{M} / 10^{18} \mathrm{~g} / \mathrm{s}\right)^{1 / 2},
$$

where $\dot{M}$ is the accretion rate. If the accretion rate is at its minimum value of $10^{15} \mathrm{~g} / \mathrm{s}$, then the minimum field strength of MSP is obtained as

$$
B_{\text {min }} \simeq 10^{7}(G)\left(\dot{M} / 10^{15} \mathrm{~g} / \mathrm{s}\right)^{1 / 2} .
$$


From the known derived fields of MSPs, we find the minimum value of field is about $10^{7.5} \mathrm{G}$ (Manchester et al. 2005), which is very close to our theoretical result. Moreover, the field and spin evolutions with the accreted mass can be given, and both decay until the bottom values after system accretes about 0.2 solar mass.

\section{Does NS field decay after the accretion phase?}

Generally, from a long-term point of views, the field decays while the accreted mass is added, and field has little decay if the accretion phase stops. The evidence for this can be found from the binary pulsar system (NS+white dwarf), where the temperature of WD is observed that implies a cooling age of system, which gives a conclusion that the field has little decay at the time scale of $10^{9} \mathrm{yr}$ if there is no accretion.

\section{The accretion must result in the field decay of NS?}

From the accretion induced field decay model (Zhang \& Kojima 2006), if the accretion MHD dragging at the polar cap is totally frozen, then all accreted matter contributes to the field decay. In case the MHD frozen efficiency is not $100 \%$, for instance $1 \%$, then 0.2 solar mass accretion only makes field decay to $10^{10} \mathrm{G}$. If this efficiency is as low as $0.01 \%$, then 0.2 solar mass accretion makes the little field decay. The frozen efficiency may be related to the magnetic inclination angle of NS, then detail of which is still in consideration. Say, some NSs may have little field decays after accreting 0.2 solar mass.

\section{The role of accretion rate on MSP field}

The accretion mass has a dominant role in MSP evolution; the accretion rate is also a factor. From X-ray NS, the average rate is about $\dot{M} / 10^{17} \mathrm{~g} / \mathrm{s}$. If the rate is less than $\dot{M} / 10^{15} \mathrm{~g} / \mathrm{s}$, then the Ohmic decay velocity will be faster than the accretion deepen-in velocity, while the accretion cannot bury the field into the NS core region. Therefore, a minimum accretion rate is needed for the field decay. The high accretion rate corresponds to a high bottom field, e.g. Eddington rate will correspond to a bottom field of $10^{8.5} \mathrm{G}$.

\section{The mass of MSP: 1.6 solar mass on average}

Two decades ago, researchers often thought that the mass of MSP should be usually as high as $2.0 M_{\odot}$. However, the recent statistics of 65 measured NS masses indicates that the average mass of MSP is only 1.6 solar mass (Zhang et al. 2011), comparing to the mass of slow rotating NS of about 1.4 solar mass, and MSP seems to absorbs only 0.2 solar mass in average. Then a question arises where it goes the one solar mass of WD companion? Does an MSP only accrete $20 \%$ mass of its companion? After the spinning-up of an MSP, some accreted mass is finally rejected from the NS? The EOS of MSPs is different from that of normal NSs? These questions are still open.

\section{Alternative possibility: MSP formation from AIC?}

MSPs are usually thought to be formed by a spin-up of NS in a binary system by accretion. Then, the idea that an MSP forms in an accretion induced collapse of a WD is not automatically excluded. What is a specialty of an MSP from AIC? The mass of an MSP by AIC should be less than Chandrasekhar limit, e.g. 1.3 solar mass. From the measured masses of MSPs (Zhang et al. 2011), it really exist 3 MSPs with the masses less 
than 1.3 solar masses. Then we cannot be sure if these low mass MSPs are formed in the low mass states, e.g. one solar mass, or by AIC processes. More MSP masses are needed, by which we can distinguish the details of MSP mass and its formation processes.

\section{Bottom magnetic field of MSP has no relation to its initial field}

The evolution history of MSPs can be understood in this way: the initial magnetic field of NS can be as high as $B \sim 10^{13} \mathrm{G}$, and it can decay to $B \sim 10^{8} \mathrm{G}$, after accreting $\sim 0.2 M_{\odot}$, while NS magnetosphere is the same size of NS. In other words, the bottom field of MSP is determined by the condition that the radius of NS magnetosphere equals the radius of NS. This fact means that the bottom field is nothing to do with its initial field strength! From the field and spin period distributions of MSPs and pulsars (Wang et al. 2011), we notice that the field distribution of normal pulsars ranges at $B \sim 10^{11-13} \mathrm{G}$, then that of MSPs ranges narrowly at $B \sim 10^{8-9} \mathrm{G}$, which should be the effect of bottom field, at where the fields stops but is no relation to its born field. The significant application of bottom field is that the high and low luminosity X-ray sources, $\mathrm{Z}$ and Atoll, share the similar $\mathrm{kHz}$ QPO frequencies, indicating the similar magnetosphere radii at scale of NS radii (Zhang 2004; Zhang et al. 2006)

\section{Magnetic structure of MSP: strong magnetic domain $10^{14-15} \mathbf{G}$ ?}

If accretion drives decays of the magnetic field of an MSP, is the magnetic structure altered too? Yes; the polar field lines of MSP are dragged to the equator region, where all field lines are sinked into the core region of NS (Zhang \& Kojima 2006). Thus, the estimation indicates that the polar field of MSP is as low as $10^{8} \mathrm{G}$, the equator field, beneath the NS surface, can be as high as $10^{13-14} \mathrm{G}$, and the core field of NS may be even higher that $10^{14} \mathrm{G}$, e.g. $10^{15} \mathrm{G}$. In other words, the MSP magnetic structure is redistributed after accretion. The leaked-out field lines of the MSP may produce the effect that the local field at the magnetic equator should be much higher than $10^{8} \mathrm{G}$, e.g. $10^{11-12} \mathrm{G}$, then the global field of MSP is dominated by a polar field with the low value $10^{8} \mathrm{G}$. Radio observations of MSPs should reflect their low field characteristic, while the $\mathrm{X}$-ray observation may show their local strong fields. The complex magnetic structure of MSP may be noticed from the high energy emissions, e.g. Fermi, in the future.

\section{Acknowledgements}

This work has been supported by National Basic Research Program of China (2012CB821800), NSFC10773017 and NSFC11173034.

\section{References}

Alpar M. A., Cheng A. F., Ruderman M. A., \& Shaham J., 1982, Nature, 300, 728

Bhattacharya D. \& van den Heuvel E. P. J. 1991, Phys. Rep., 203, 1

Lyne A. G., Burgay M. \& Kramer, M. et al., 2004, Science, 303, 1153.

Manchester R. N., Hobbs G. B., Teoh A., \& Hobbs M. , 2005, AJ, 129, 1993

Tauris M., 2012, Science, 335, 561

van den Heuvel E. P. J., 2004, Science, 303, 20

Wang, J., Zhang, C. M., Zhao, Y. H. et al., 2011, A\&A, 526, 88

Wijnands R. \& van der Klis M., 1998, Nature, 394, 344

Zhang, C. M. 2004, A\&A, 423, 401

Zhang C. M., Kojima Y., 2006, MNRAS, 336, 137

Zhang, C. M., Yin, H. X., Zhao, Y. H. et al. 2006, MNRAS, 366, 1373

Zhang C. M., Wang J., Zhao Y.H. et al., 2011, A\& A, 527, 83 